\title{
Factors Moderating the Impact of After Death Communications on Beliefs and Spirituality
}

Left running head: OMEGA—Journal of Death and Dying

Short title : Penberthy et al.

Jennifer K. Penberthy ${ }^{1}$ Marieta Pehlivanova ${ }^{1}$ (D) Tevfik Kalelioglu ${ }^{1}$ Chris A. Roe ${ }^{2}$ CallumE. Cooper ${ }^{2}$ David Lorimer ${ }^{3}$ Evelyn Elsaesser ${ }^{4}$

${ }^{1}$ Department of Psychiatry and Neurobehavioral Sciences, 2358University of Virginia, University of Virginia, Charlottesville, United States

${ }^{2}$ Centre for Psychology \& Social Sciences, 6087The University of Northampton, The University of Northampton, Northampton, United Kingdom

${ }^{3}$ Independent Researcher, Colombe sur l'Hers, France

${ }^{4}$ Independent Researcher, Chavannes de Bogis, Switzerland

Tevfik Kalelioglu, Department of Psychiatry \& Neurobehavioral Sciences, University of Virginia, Charlottesville, VA, United States.Email: tevfikkaleli@hotmail.com

\begin{abstract}
After death communications(ADCs) are defined as perceived spontaneous contacts with living individuals by the deceased. This research presents on a subset of data from a recent large international survey of individuals who experienced ADCs and provided systematic information regarding these experiences. In our research we explore the impact of having an ADC on reported spirituality, religiosity, beliefs and attitudes about death and dying and also explore the moderating factors of this impact. We found that having an ADC was perceived as a positive life experience and that it was associated with a reduction in fear of death, belief in life after death and that the deceased could communicate with the living, and increased reported spirituality. Moderating factors include aspects of having or desiring physical contact with the deceased as well as perceiving some emotional reaction to the ADCs. Future directions for research exploration are also provided based on our findings.
\end{abstract}

Keywords: death, spirituality, religiosity, fear of death, after death communication 


\section{Introduction}

Experts have defined after death communication (ADC) in a variety of ways (Guggenheim \& Guggenh eim, 1995; Houck, 2005; La Grande, 1997; Long, 1999). Generally, an ADC is defined as a spontaneous phenomenon in which a living individual has a feeling or sense of direct contact with a deceased person. These contacts or communications are direct in that they are not mediated by other persons, such as psychics and mediums, or by utilizing particular rituals or devices specifically intended to enable contact with the deceased, and spontaneous in that they are not solicited by the recipients. ADCs can occur in a multitude of forms and situations as well as in a waking or sleeping state. ADCs are reported as occurring in different forms and levels of intensity, with a variety of associated sensations, including vision, taste, hearing, smell, and feeling both physical presence, such as touch or pressure, as well as a sense of the presence of the deceased. ADCs occur across cultures, race, age, socio-economic status, educational level, gender, and religious beliefs (Streit-Horn, 2013).

ADCs occur more frequently than one may imagine, with an estimated $25-50 \%$ of mourners worldwide having experienced one or more spontaneous ADCs (Guggenheim \& Guggenheim, 1995; L a Grande, 1997). According to a European Values Study, 127 million or 25\% of Europeans report experiencing an ADC, and $20-40 \%$ of Americans report experiencing an ADC in their lifetime (Harald sson, 2006). Other surveys of persons, in particular mourners, report that between $50 \%$ and $60 \%$ had one or several ADCs (Burton, 1982; Castelnovo et al., 2015; Haraldsson, 2012; Kalish \& Reynolds, 1 973) Overall, testimonies collected in different countries and over the last centuries suggest this phenomenon to be fairly universal and timeless (Guggenheim \& Guggenheim, 1995). Additionally, experts in the fields of grief, bereavement, counseling, and palliative care support the idea that ADCs are common, natural experiences that most individuals find comforting, encouraging, and sometimes even life-saving (Arcangel, 2005; Devers, 1997; Guggenheim \& Guggenheim, 1995; La Grande, 1997, 1999; Long, 1999).

Despite their seemingly widespread occurrence, we know little about who is most likely to experience an ADC or why or when. We also know very little about the impact of an ADC on the individual experiencing it. What little research does exist, appears to support the idea that the emotional impacts and interpretations of ADCs can vary across individuals. AQ1 For example, research by Datson-Mar wit et al. (1997) demonstrated that $86 \%$ of their participants who reported having an ADC characterized the experience as comforting, while $6 \%$ found it upsetting, and $8 \%$ found it frightening $t$ Đatson \& Marwit, 1997). Individuals who have experienced ADCs have reported a decrease in negative feelings such as sadness, grief, longing, incomplete communication, regret, and remorse, as well as an increase in positive feelings of understanding, appreciation, gratitude, love, and forgiveness ( Hastings, 2012). Death anxiety has tended to be lower among individuals who reported having contact with a deceased loved one. However, the data further reveal that this potential benefit was likely to 
occur only among older people who interpreted ADCs from a religious perspective and that these experiences were also likely to have strengthened their existing religious beliefs (Krause, 2011). Kwilec ki (2011) reported that having an ADC tended to reinforce comforting religious beliefs, including assurances of a fulfilling afterlife, as well as increased positive explanations of unexpected deaths (Kwil ecki, 2011). Likewise, Keen et al. (2013) evaluated individuals who reported ADCs and found that the sense of presence of a deceased loved one was reported as comforting. However, those individuals who admitted they were struggling to make sense of a loss tended to perceive ADCs as frightening (Ke en et al., 2013). Steffen and Coyle (2011) identified similar themes in a study of 12 participants who reported sense-of-presence ADCs and reported that although participants described the experiences as almost exclusively beneficial, the accounts often revealed a struggle with culturally available understandings that created cognitive dissonance in some (Steffen \& Coyle, 2011).

In the study conducted by Streit-Horn (2013) participants frequently endorsed that the experience of an ADC was followed by increased feelings of reassurance and comfort. This comfort was attributed to their associated newfound knowledge of the continued existence of the deceased individual following bodily death and of an ongoing relationship with the deceased (Streit-Horn, 2013). In a study that evaluated ADCs in parents of deceased children, the majority of the parents reported that their beliefs about life after death had changed since their child's death (Sormanti \& August, 1997). In the same study, the parents reported a strengthening of existing beliefs, several reported having new beliefs, and some found comfort, claiming that their experiences confirmed that their child was in a safe place with other deceased family members or with God. For them, this was confirmation of an after-life (Sormanti \& August, 1997).

Whatever the ontological status of ADCs might be, they are perceived as real and significant by a great number of individuals who experience them, and therefore certainly deserve their place on the consciousness research agenda. Existing research does appear to provide some support for the claim that experiencing an ADC can have a positive impact and may even change beliefs. This is not, however, a universal finding, and further research is needed to identify factors that contribute to whether ADCs are perceived as positive or negative. In this current study, we wish to map the impacts reported by people who have experienced an ADC, including religious and spiritual beliefs, as well as beliefs about the afterlife and deceased individuals contacting the living, and explore the factors that moderate such effects. Streit-Horn (2013) found a common theme of reticence among participants to report their ADCs (Streit-Horn, 2013).; many endorsed reluctance to share their ADCs for fear of being judged, ridiculed, and/or stigmatized (Amatuzio \& Matthews, 2007; Ring, 2008). This finding possibly indicates that ADCs may be underreported. For example, Olson et al. (1985) reported that $54 \%$ of study participants had never told anyone about their ADCs prior to their being interviewed for the research study (Olson et al., 1985).

Several ADC researchers, particularly those who conducted interviews, reported that participants 
expressed relief in having an opportunity to discuss their ADCs, and many reported positive impacts of both the ADC and sharing it (Beischel et al., 2015; Guggenheim \& Guggenheim, 1995; Keen et al., 20 13) Thus, an improved understanding of the phenomenology and impact of ADCs is important not only for research but also to disseminate knowledge about the prevalence of such experiences and potentially reduce stigma, so as to encourage open discussion and self reflection (Steffen \& Coyle, 201 1). Additionally, increased knowledge about ADCs is important for the public at large, since these experiences may provide a new perspective of death and life.

Our work is a small part of a larger international research project that aims to explore the phenomenology, and impact of ACDs more fully in several countries. The study was designed, data eolleetion was eompleted and the dataset was analyzed by the projeet team of Evelyn Elsaesser, Chris A. Roe, and Callum E. Cooper at the University of Northampton, UK, with David Lorimer as advisor (Elsaesser et al., 2021) . While a primary objective of the larger overall project is to gain a better understanding of the phenomenology and the impact of spontaneous ADCs, in this paper, we will focus on the impact of ADCs on beliefs and spirituality and attitudes about death and dying, as well as the factors that moderate such changes. Research questions focus on exploring not only the selfreported changes in beliefs and attitudes in individuals who experienced an ADC, but also investigating moderating factors of this impact.

\section{Materials and Methods}

\section{Procedure}

This research involved online data collection between February 2018 and January 2020 in the three languages of the project, namely in English, French and Spanish. The survey included 194-items and was completed by participants of several European countries, Latin American countries and the United States of America. The research project recruited adults only and received ethical approval from the University of Northampton (ref: FHSRECSS00084) and wapre-registered (ref: KPU Registry 1046) with the Koestler Unit Study registry at the University of Edinburgh (https://koestlerunit.wordpress.com/study-registry/registered-studies/). The survey landing page reminded participants of the nature of the study and of what participation would entail, including that data were volunteered anonymously so it was not possible to withdraw data once submitted. Respondents confirmed in writing that they consented to participate in order to progress to the questionnaire. Participants were recruited using a purposive snowball sampling method by advertising the survey during public talks and through social media forums that specialize in ADCs and related phenomena. Interested parties were referred to the Principal Investigator's web page that gave further information about the project and provided a link to the survey. Each survey version was made 'live' for pre-specified periods of time - the English version from 9 August 2018 - 31 January 2019, the French version from 15 September 2018 - 31 March 2019, and the Spanish version from 21 October 
2018 - 30 April 2019. Participants completed the survey in their own time. There was no facility to save progress so the whole questionnaire needed to be completed in a single session (though the web link would not time out and so could be left open for as long as required). Participants needed to complete the whole measure for their data to be submitted for subsequent analysis. Voluntary participants were oriented to the project, consented, and invited to answer a one-time online survey about any ADCs they had experienced. Data was collected and stored on a secure,passwordprotected network with only the Principle Investigators having access in order to help protect the security of the data. Participants also provided demographics and socioeconomic characteristics. Nine hundred and ninety-one participants completed both the consent form and the questionnaire. The main themes of the questionnaires were: circumstances of occurrence of ADC, type of ADC, message conveyed in the $\mathrm{ADC}$, emotions associated with $\mathrm{ADC}$, sense of reality associated with the experience, impact and implications for the grieving process, profile of the recipient, and profile of the deceased person perceived (including cause of death). Quantitative research findings of the complete data set have been summarized and are in submission for publication. Qualitative analyses on specific themes have been or will be published individually (Woollacott et al., 2021). In this current manuscript, we report the impact of specific variables on changes in beliefs and spirituality in those reporting ADCs and explore moderating factors on such changes.

\section{Statistical Analysis}

To assess change in beliefs pre- and post-ADC, we calculated the difference between beliefs at both time points, such that higher difference indicates increased beliefs after the ADC. The change in beliefs was assessed using a paired-sample t-test. For the purpose of calculating belief changes for variables assessed on a Yes/No/Unsure scale, "Yes" was given a value of 2, "Unsure" was given a value of 1 , and "No" was given a value of 0 .

We used ordinal and binary logistic regression models to investigate factors which predict changes in beliefs from pre- to post-ADC. Initially, each independent variable was considered in a separate univariate model predicting the change outcome. If an individual variable reached level of significance of 0.10 or below, it was subsequently included in a multiple logistic regression model, simultaneously including (and controlling for) all predictors with $p \leq 0.10$. Thus, the p-values obtained from multiple regression models show statistical significance for each variable after adjusting for all other variables in the model. For categorical variables, one category was chosen as a 'reference' category, and comparisons for all other categories against the reference are reported. For religious and spiritual beliefs, change was modeled as a dependent variable with three ordinal levels: 3 = beliefs increased; 2 = beliefs remained the same; 1 = beliefs decreased. For beliefs in life after death and the ability of deceased people to contact the living, change was modeled as a binary dependent variable: $1=$ beliefs strengthened; $0=$ beliefs remained the same or weakened. In the latter case, the choice of a binary dependent variable was made for ease of interpretation and for practical reasons since there were very 
few individuals who weakened their beliefs in life after death or the ability of the deceased to contact the living. In all analyses, the models predicted an increase in beliefs post-ADC.

In both univariate and multivariate analyses, the strength of association between a factor and the outcome of interest was expressed as an odds ratio $(O R)$ and its corresponding $95 \%$ confidence interval (CI). An $O R$ of 1 indicates no effect, such that, for categorical factors, increased beliefs are not more likely in either of the categories being compared, while values greater or less than 1 indicate that the category of interest is associated with higher or lower odds of the outcome, respectively.

A p-value was taken as significant if less than 0.05. All statistical analyses were conducted in SAS 9.4.

\section{Results}

Nine hundred ninety one individuals who reported on their experienced ADCs were included in the study. The overall ADC group consisted of 143 males (14.5\%), 842 females (84.9\%), and 4 others $(0.4 \%)$. The mean age of participants was $51.19 \pm 13.26$ years (min: 18 max: 89 ). Four hundred and twelve were English speaking (41.6\%), 434 were French speaking (43.7\%) and 145 were Spanish speaking (14.7\%). Demographic information is summarized in Table 1.

Table 1. Descriptive Statistics and Probable Moderating Factors for Samples of CORTs.

\begin{tabular}{|l|l|l|}
\hline Variable & $N^{\text {a }}$ & Mean \pm SD or count (\%) \\
\hline Age & 981 & $51.19 \pm 13.26$ \\
\hline Sex (female) & 981 & 84.9 \\
\hline Education & 986 & 47.8 \\
\hline University & 475 & 17.5 \\
\hline College or equivalent & 174 & 22.2 \\
\hline Secondary level-university & 220 & 11.8 \\
\hline Secondary school & 117 & 80 \\
\hline Multiple ADCs (yes) & 990 & 47.5 \\
\hline Physical contact (yes) & 982 & 46.2 \\
\hline Saw deceased (yes) & 989 & 43.3 \\
\hline Heard deceased (yes) & 986 & 13.2 \\
\hline Touched deceased (yes) & 498 & 27.6 \\
\hline Smelled deceased (yes) & 982 & 58.6 \\
\hline Received message (yes) & 981 & 34.1 \\
\hline Sensed presence of deceased (yes) & 987 & \\
\hline
\end{tabular}




\begin{tabular}{|l|l|l|}
\hline Variable & $N^{\mathrm{a}}$ & Mean \pm SD or count (\%) \\
\hline Crisis ADC (yes) & 988 & 20.7 \\
\hline Frightened by ADC (yes) & 987 & 12.1 \\
\hline Sentiment about ADC (positive) & 987 & 91 \\
\hline Knew the deceased (yes) & 982 & 90.2 \\
\hline Comfort from ADC (yes) & 907 & 73 \\
\hline
\end{tabular}

${ }^{\mathrm{a}} \mathrm{N}$ is number of observations for each variable in each sample.

We examined our hypotheses that experiencing an ADC would be perceived as a positive life experience in the majority of participants and found this to be supported. Nearly one-third of participants considered their perceived ADC as 'life changing' and 57.8\% endorsed that their ADC was 'moderately important' or 'important'. We further hypothesized that having an ADC would be associated with reduced fear of death. This hypothesis was supported. Following the perceived ADC, $31 \%(n=308)$ of participants defined their fear of death as decreased, 29.2\% $(n=290)$ reported their fear of death had disappeared, while 32.8\% $(n=326)$ described no change in their fear of death, and only $0.8 \%(n=8)$ mentioned an increase.

We also hypothesized that experiencing an ADC would be associated with increased beliefs in life after death as well as other spiritual or religious beliefs, and this was only partially supported. There was a statistically significant increase in participants regarding endorsed spirituality ( $t=18.94, p<0.001$ ) but not in being religious $(t=0.371, p=0.710)$ after experiencing ADC (Table 2). Overall, there was a significant change observed regarding the belief in life after death $(t=16.26, p<0.001)$ and the belief that the deceased can communicate with living people. $(t=19.68, p<0.001)$ (see Table 2$)$.

\section{Table 2. Effect of ADC on Changes in Religiosity, Spirituality, Life After Death and Deceased Can Contact Living Persons.}

\begin{tabular}{|l|l|l|l|l|l|l|}
\hline Variable & Na & Mean \pm SD & Median & Min and max & t & p \\
\hline $\begin{array}{l}\text { Change in } \\
\text { religiosity }\end{array}$ & 983 & $0.01 \pm 0.77$ & 0 & -4 and 4 & 0.37 & 0.71 \\
\hline $\begin{array}{l}\text { Change in } \\
\text { spirituality }\end{array}$ & 986 & $0.57 \pm 0.94$ & 0 & -3 and 4 & 18.95 & $<.0001$ \\
\hline $\begin{array}{l}\text { Change in } \\
\text { belief about life } \\
\text { after death }\end{array}$ & 984 & $0.34 \pm 0.65$ & 0 & -2 and 2 & 16.27 & $<.0001$ \\
\hline
\end{tabular}




\begin{tabular}{|c|c|c|c|c|c|c|}
\hline Variable & $\mathrm{Na}$ & Mean \pm SD & Median & Min and max & t & p \\
\hline $\begin{array}{lr}\text { Change } & \text { in } \\
\text { belief } & \text { about } \\
\text { deceased } & \text { can } \\
\text { contact } & \text { living } \\
\text { persons } & \end{array}$ & 987 & $0.45 \pm 0.72$ & 0 & -2 and 2 & 19.69 & $<.0001$ \\
\hline
\end{tabular}

${ }^{\mathrm{a}} \mathrm{N}$ is number of observations for each variable in each sample.

We also wished to examine, in an exploratory fashion, the moderating factors for such changes. Probable moderating factors are shown in Table 1. As a first step, we explored predictors of changes in beliefs pre- and post-ADC in univariate logistic regressions. In the follow-up multivariate analyses, we included variables with a $\mathrm{p}$ value $<0.1$ in univariate analysis as candidate predictors. All multivariate results report effects controlling for other variables. Multivariate analysis revealed that having multiple ADCs was the only moderating factor that was related to the change in religious beliefs (see Table 4). Specifically, participants with multiple ADCs showed an almost $50 \%$ increase in the odds of endorsing increased religious beliefs after ADC, compared with individuals who did not report multiple ADCs.

Table 3. Univariate Analyses Predicting Change in Religious Beliefs Pre- and Post-ADC (Ordinal Logistic Regression).

\begin{tabular}{|l|l|l|l|l|l|l|}
\hline Predictor & $N$ & $\begin{array}{l}\text { Category } \\
\text { (reference) }\end{array}$ & OR & Wald & $p$ & $95 \%$ CI for OR \\
\hline Age & 971 & - & 0.99 & 1.22 & .27 & {$[0.98,1.005]$} \\
\hline Sex & 977 & Female (male) & 1.51 & 4.08 & .04 & {$[1.01,2.25]$} \\
\hline Education & 978 & - & 1.13 & 3.41 & .07 & {$[0.99,1.28]$} \\
\hline Multiple ADCs & 981 & $\begin{array}{l}\text { Yes } \\
\text { (no/unsure) }\end{array}$ & 1.43 & 3.84 & .05 & {$[1,00,2.03]$} \\
\hline $\begin{array}{l}\text { Physical } \\
\text { contact }\end{array}$ & 972 & $\begin{array}{l}\text { Yes } \\
\text { (no/unsure) }\end{array}$ & 1.31 & 3.46 & .06 & {$[0.99,1.74]$} \\
\hline Saw deceased & 979 & $\begin{array}{l}\text { Yes } \\
\text { (no/unsure) }\end{array}$ & 0.94 & 0.18 & .67 & {$[0.71,1.25]$} \\
\hline $\begin{array}{l}\text { Heard } \\
\text { deceased }\end{array}$ & 976 & $\begin{array}{l}\text { Yes } \\
\text { (no/unsure) }\end{array}$ & 0.78 & 2.83 & .09 & {$[0.59,1.04]$} \\
\hline $\begin{array}{l}\text { Touched } \\
\text { deceased }\end{array}$ & 493 & $\begin{array}{l}\text { Yes } \\
\text { (no/unsure) }\end{array}$ & 1.05 & 0.06 & .08 & {$[0.68,1.65]$} \\
\hline $\begin{array}{l}\text { Smelled } \\
\text { deceased }\end{array}$ & 972 & $\begin{array}{l}\text { Yes } \\
\text { (no/unsure) }\end{array}$ & 1.37 & 3.85 & .04 & {$[1.00,1.88]$} \\
\hline $\begin{array}{l}\text { Received } \\
\text { message }\end{array}$ & 972 & $\begin{array}{l}\text { Yes } \\
\text { (no/unsure) }\end{array}$ & 0.85 & 1.32 & .25 & {$[0.63,1.13]$} \\
\hline
\end{tabular}




\begin{tabular}{|c|c|c|c|c|c|c|}
\hline Predictor & $N$ & $\begin{array}{l}\text { Category } \\
\text { (reference) }\end{array}$ & OR & Wald $_{1}$ & $p$ & $95 \% \mathrm{CI}$ for $O R$ \\
\hline $\begin{array}{l}\text { Sensed } \\
\text { pres e n c e of } \\
\text { deceased }\end{array}$ & 977 & $\begin{array}{l}\text { Yes } \\
\text { (no/unsure) }\end{array}$ & 0.89 & 0.51 & .47 & {$[0.66,1.20]$} \\
\hline Crisis ADC & 978 & $\begin{array}{l}\text { Yes } \\
\text { (no/unsure) }\end{array}$ & 0.91 & 0.26 & .60 & {$[0.64,1.29]$} \\
\hline $\begin{array}{l}\text { Frightened by } \\
\text { ADC }\end{array}$ & 977 & $\begin{array}{l}\text { Yes } \\
\text { (no/unsure) }\end{array}$ & 1.07 & 0.12 & .72 & {$[0.70,1.65]$} \\
\hline $\begin{array}{l}\text { Sentiment about } \\
\text { ADC }\end{array}$ & 977 & $\begin{array}{l}\text { Positive } \\
\text { (negative/neutral) }\end{array}$ & 0.71 & 1.67 & .19 & {$[0.43,1.18]$} \\
\hline $\begin{array}{l}\text { Knew the } \\
\text { deceased }\end{array}$ & 972 & $\begin{array}{l}\text { Yes } \\
\text { (no/unsure) }\end{array}$ & 1.62 & 3.73 & .05 & {$[0.99,2.66]$} \\
\hline $\begin{array}{l}\text { Comfort from } \\
\text { ADC }\end{array}$ & 978 & $\begin{array}{l}\text { Yes } \\
\text { (no/unsure/N/A) }\end{array}$ & 1.10 & 0.40 & .052 & {$[0.80,1.52]$} \\
\hline
\end{tabular}

$O R=$ odds ratio; Wald $_{1}=$ Wald statistic with 1 degree of freedom; $p=\mathrm{p}$-value; $\mathrm{CI}=$ confidence interval .

Table 4. Multivariate Analysis Predicting Change in Religious Beliefs Pre- and Post-ADC (Ordinal Logistic Regression), $\mathbf{N}=939$.

\begin{tabular}{|l|l|l|l|l|l|}
\hline Predictor & $\begin{array}{l}\text { Category } \\
\text { (reference) }\end{array}$ & OR & Wald 1 & $p$ & $95 \%$ CI for OR \\
\hline Sex & Female (male) & 1.49 & 3.71 & 0.05 & {$[0.99,2.25]$} \\
\hline Education & - & 1.10 & 2.13 & 0.14 & {$[0.96,1.26]$} \\
\hline Multiple ADCs & Yes (no/unsure) & 1.49 & 4.36 & 0.03 & {$[1.02,2.16]$} \\
\hline Physical contact & Yes (no/unsure) & 1.26 & 2.42 & 0.11 & {$[0.94,1.71]$} \\
\hline Heard deceased & Yes (no/unsure) & 0.77 & 2.85 & 0.09 & {$[0.57,1.04]$} \\
\hline Smelled deceased & Yes (no/unsure) & 1.15 & 0.72 & 0.39 & {$[0.83,1.59]$} \\
\hline $\begin{array}{l}\text { Knew the } \\
\text { deceased }\end{array}$ & Yes (no/unsure) & 1.54 & 2.83 & 0.09 & {$[0.93,2.57]$} \\
\hline
\end{tabular}

$O R=$ odds ratio; Wald $_{1}=$ Wald statistic with 1 degree of freedom; $p=\mathrm{p}$-value; $\mathrm{CI}=$ confidence interval.

Table 5. Univariate Analyses Predicting Change in Spiritual Beliefs Pre- and Post-ADC (Ordinal Logistic Regression).

\begin{tabular}{|l|l|l|l|l|l|l|}
\hline Pre dictor & $\boldsymbol{N}$ & $\begin{array}{l}\text { Category } \\
\text { (reference) }\end{array}$ & OR & Wald $_{1}$ & $\boldsymbol{p}$ & $95 \%$ CI for OR \\
\hline Age & 974 & - & 0.99 & 0.38 & .53 & {$[0.98,1.01]$} \\
\hline
\end{tabular}




\begin{tabular}{|c|c|c|c|c|c|c|}
\hline Pre dictor & $N$ & $\begin{array}{l}\text { Category } \\
\text { (reference) }\end{array}$ & $O R$ & Wald $_{1}$ & $p$ & $95 \%$ CI for $O R$ \\
\hline Sex & 980 & Female (male) & $0.98 \theta 4$ & 0.00 & .94 & {$[0.68,1.41]$} \\
\hline Education & 981 & - & 1.03 & 0.00 & .95 & {$[0.89,1.12]$} \\
\hline Multiple ADCs & 984 & $\begin{array}{l}\text { Yes } \\
\text { (no/unsure) }\end{array}$ & 1.27 & 2.16 & .14 & {$[0.92,1.76]$} \\
\hline $\begin{array}{l}\text { Physical } \\
\text { contact }\end{array}$ & 975 & $\begin{array}{l}\text { Yes } \\
\text { (no/unsure) }\end{array}$ & 1.52 & 10.18 & .00 & {$[1.17,1.96]$} \\
\hline Saw deceased & 982 & $\begin{array}{l}\text { Yes } \\
\text { (no/unsure) }\end{array}$ & 1.07 & 0.29 & .59 & {$[0.83,1.38]$} \\
\hline $\begin{array}{l}\text { Heard } \\
\text { deceased }\end{array}$ & 979 & $\begin{array}{l}\text { Yes } \\
\text { (no/unsure) }\end{array}$ & 0.95 & 0.14 & .70 & {$[0.73,1.23]$} \\
\hline $\begin{array}{l}\text { Touched } \\
\text { deceased }\end{array}$ & 494 & $\begin{array}{l}\text { Yes } \\
\text { (no/unsure) }\end{array}$ & 1.21 & 0.90 & .34 & {$[0.81,1.82]$} \\
\hline $\begin{array}{l}\text { Smelled } \\
\text { deceased }\end{array}$ & 975 & $\begin{array}{l}\text { Yes } \\
\text { (no/unsure) }\end{array}$ & 0.99 & 0.00 & .97 & {$[0.74,1.32]$} \\
\hline $\begin{array}{l}\text { Received } \\
\text { message }\end{array}$ & 975 & $\begin{array}{l}\text { Yes } \\
\text { (no/unsure) }\end{array}$ & 0.84 & 1.52 & .21 & {$[0.65,1.10]$} \\
\hline $\begin{array}{l}\text { Sensed } \\
\text { pres e n c e of } \\
\text { deceased }\end{array}$ & 980 & $\begin{array}{l}\text { Yes } \\
\text { (no/unsure) }\end{array}$ & 0.91 & 0.41 & .51 & {$[0.69,1.19]$} \\
\hline Crisis ADC & 981 & $\begin{array}{l}\text { Yes } \\
\text { (no/unsure) }\end{array}$ & 0.76 & 2.80 & .09 & {$[0.55,1.04]$} \\
\hline $\begin{array}{l}\text { Frightened by } \\
\text { ADC }\end{array}$ & 980 & $\begin{array}{l}\text { Yes } \\
\text { (no/unsure) }\end{array}$ & 1.28 & 1.58 & .20 & {$[0.87,1.88]$} \\
\hline $\begin{array}{l}\text { Sentiment about } \\
\text { ADC }\end{array}$ & 980 & $\begin{array}{l}\text { Positive } \\
\text { (negative/neutral) }\end{array}$ & 1.41 & 1.98 & .15 & {$[0.87,2.28]$} \\
\hline $\begin{array}{l}\text { Knew the } \\
\text { deceased }\end{array}$ & 975 & $\begin{array}{l}\text { Yes } \\
\text { (no/unsure) }\end{array}$ & 0.85 & 0.48 & .48 & {$[0.54,1.33]$} \\
\hline $\begin{array}{l}\text { Comfort from } \\
\text { ADC }\end{array}$ & 981 & $\begin{array}{l}\text { Yes } \\
\text { (no/unsure/N/A) }\end{array}$ & 1.43 & 5.69 & .02 & {$[1.06,1.92]$} \\
\hline
\end{tabular}

$O R=$ Odds ratio; Wald $_{1}=$ Wald statistic with 1 degree of freedom; $p=$ p-value; $\mathrm{CI}=$ confidence interval.

Table 6. Multivariate Analysis Predicting Change in Spiritual Beliefs Pre- and Post-ADC (Ordinal Logistic Regression), $\mathrm{N}=967$.

\begin{tabular}{|l|l|l|l|l|l|}
\hline Predictor & $\begin{array}{l}\text { Category } \\
\text { (reference) }\end{array}$ & OR & Wald 1 & $p$ & $95 \%$ CI for OR \\
\hline Physical contact & Yes (no/unsure) & 1.51 & 9.60 & 0.002 & {$[1.16,1.96]$} \\
\hline Crisis ADC & Yes (no/unsure) & 0.72 & 3.67 & 0.05 & {$[0.52,1.00]$} \\
\hline
\end{tabular}




\begin{tabular}{|c|c|c|c|c|c|}
\hline Pre dictor & $\begin{array}{l}\text { Category } \\
\text { (reference) }\end{array}$ & OR & Wald $_{1}$ & $p$ & $95 \%$ CI for $O R$ \\
\hline $\begin{array}{l}\text { Comfort from } \\
\text { ADC }\end{array}$ & $\begin{array}{l}\text { Yes } \\
\text { (no/unsure/N/A) }\end{array}$ & 1.35 & 3.81 & 0.05 & {$[0.99,1.82]$} \\
\hline
\end{tabular}

$O R=$ Odds ratio; Wald $_{1}=$ Wald statistic with 1 degree of freedom; $p=$ p-value; $\mathrm{CI}=$ confidence interval .

Table 7. Univariate Analyses Predicting Beliefs About Life After Death Pre- and PostADC (Binary Logistic Regression).

\begin{tabular}{|c|c|c|c|c|c|c|}
\hline Pre dictor & $N$ & $\begin{array}{l}\text { Category } \\
\text { (reference) }\end{array}$ & OR & Wald $_{1}$ & $p$ & $95 \%$ CI for $O R$ \\
\hline Age & 972 & - & 0.99 & 0.01 & .91 & {$[0.98,1.01]$} \\
\hline Sex & 978 & Female (male) & 1.18 & 0.63 & .42 & {$[0.78,1.79]$} \\
\hline Education & 977 & - & 0.87 & 6.54 & .01 & {$[0.73,0.95]$} \\
\hline Multiple ADCs & 981 & $\begin{array}{l}\text { Yes } \\
\text { (no/unsure) }\end{array}$ & 1.02 & 0.01 & .91 & {$[0.71,1.48]$} \\
\hline $\begin{array}{l}\text { Physical } \\
\text { contact }\end{array}$ & 973 & $\begin{array}{l}\text { Yes } \\
\text { (no/unsure) }\end{array}$ & 1.08 & 0.32 & .56 & {$[0.81,1.44]$} \\
\hline Saw deceased & 980 & $\begin{array}{l}\text { Yes } \\
\text { (no/unsure) }\end{array}$ & 1.03 & 0.05 & .82 & {$[0.77,1.37]$} \\
\hline $\begin{array}{l}\text { Heard } \\
\text { deceased }\end{array}$ & 977 & $\begin{array}{l}\text { Yes } \\
\text { (no/unsure) }\end{array}$ & 0.85 & 1.20 & .27 & {$[0.63,1.13]$} \\
\hline $\begin{array}{l}\text { Touched } \\
\text { deceased }\end{array}$ & 494 & $\begin{array}{l}\text { Yes } \\
\text { (no/unsure) }\end{array}$ & 1.57 & 4.12 & .04 & {$[1.01,2.43]$} \\
\hline \begin{tabular}{|l|} 
Smelled \\
deceased
\end{tabular} & 973 & $\begin{array}{l}\text { Yes } \\
\text { (no/unsure) }\end{array}$ & 1.07 & 0.17 & .67 & {$[0.78,1.46]$} \\
\hline $\begin{array}{l}\text { Received } \\
\text { message }\end{array}$ & 973 & $\begin{array}{l}\text { Yes } \\
\text { (no/unsure) }\end{array}$ & 0.79 & 2.35 & .12 & {$[0.59,1.06]$} \\
\hline $\begin{array}{l}\text { Sensed } \\
\text { pr e s e n c e of } \\
\text { deceased }\end{array}$ & 978 & $\begin{array}{l}\text { Yes } \\
\text { (no/unsure) }\end{array}$ & 0.91 & 0.35 & .54 & {$[0.67,1.23]$} \\
\hline Crisis ADC & 979 & $\begin{array}{l}\text { Yes } \\
\text { (no/unsure) }\end{array}$ & 0.75 & 2.35 & .12 & {$[0.52,1.08]$} \\
\hline \begin{tabular}{|l|} 
Frightened by \\
ADC
\end{tabular} & 979 & $\begin{array}{l}\text { Yes } \\
\text { (no/unsure) }\end{array}$ & 2.02 & 12.10 & .00 & {$[1.36,3.01]$} \\
\hline $\begin{array}{l}\text { Sentiment about } \\
\text { ADC }\end{array}$ & 979 & $\begin{array}{l}\text { Positive } \\
\text { (negative/neutral) }\end{array}$ & 1.08 & 0.09 & .75 & {$[0.64,1.84]$} \\
\hline $\begin{array}{l}\text { Knew } \\
\text { deceased }\end{array}$ & 974 & $\begin{array}{l}\text { Yes } \\
\text { (no/unsure) }\end{array}$ & 0.96 & 0.02 & .88 & {$[0.58,1.59]$} \\
\hline $\begin{array}{l}\text { Comfort from } \\
\text { ADC }\end{array}$ & 981 & $\begin{array}{l}\text { Yes } \\
\text { (no/unsure/N/A) }\end{array}$ & 1.39 & 3.78 & .05 & {$[0.99,1.95]$} \\
\hline
\end{tabular}


$O R=$ Odds ratio; Wald $_{1}=$ Wald statistic with 1 degree of freedom; $p=$ p-value; $\mathrm{CI}=$ confidence interval .

Table 8. Multivariate Analysis Predicting Beliefs About Life After Death Pre- and PostADC (Binary Logistic Regression), $N=484$.

\begin{tabular}{|l|l|l|l|l|l|}
\hline Predictor & $\begin{array}{l}\text { Category } \\
\text { (reference) }\end{array}$ & OR & Wald 1 & $p$ & $95 \%$ CI for OR \\
\hline Education & - & 0.89 & 1.39 & 0.23 & {$[0.73,1.07]$} \\
\hline Touched deceased & Yes (no/unsure) & 1.89 & 7.24 & 0.01 & {$[1.18,3.00]$} \\
\hline Received message & Yes (no/unsure) & 0.59 & 5.53 & 0.02 & {$[0.38,0.91]$} \\
\hline $\begin{array}{l}\text { Frightened by } \\
\text { ADC }\end{array}$ & Yes (no/unsure) & 2.57 & 10.83 & 0.001 & {$[1.46,4.51]$} \\
\hline $\begin{array}{l}\text { Comfort from } \\
\text { ADC }\end{array}$ & $\begin{array}{l}\text { Yes } \\
\text { (no/unsure/N/A) }\end{array}$ & 1.21 & 0.55 & 0.45 & {$[0.73,2.01]$} \\
\hline
\end{tabular}

$O R=$ Odds ratio; Wald $_{1}=$ Wald statistic with 1 degree of freedom; $p=$ p-value; $\mathrm{CI}=$ confidence interval .

Table 9. Univariate Analyses Predicting Beliefs About Deceased Persons Can Contact the Living Persons Pre- and Post-ADC (Binary Logistic Regression).

\begin{tabular}{|l|l|l|l|l|l|l|}
\hline Predictor & $N$ & $\begin{array}{l}\text { Category } \\
\text { (reference) }\end{array}$ & OR & Wald & $p$ & $95 \%$ CI for OR \\
\hline Age & 975 & - & 0.99 & 0.74 & .38 & {$[0.98,1.01]$} \\
\hline Sex & 981 & Female (male) & 1.07 & 0.12 & .71 & {$[0.73,1.57]$} \\
\hline Education & 980 & - & 0.82 & 9.09 & .003 & {$[0.72,0.93]$} \\
\hline Multiple ADCs & 984 & $\begin{array}{l}\text { Yes } \\
\text { (no/unsure) }\end{array}$ & 0.75 & 2.78 & .09 & {$[0.54,1.04]$} \\
\hline $\begin{array}{l}\text { Physical } \\
\text { contact }\end{array}$ & 976 & $\begin{array}{l}\text { Yes } \\
\text { (no/unsure) }\end{array}$ & 1.02 & 0.02 & .87 & {$[0.78,1.33]$} \\
\hline $\begin{array}{l}\text { Saw deceased } \\
\text { Yes }\end{array}$ & 983 & 0.91 & 0.41 & .51 & {$[0.70,1.19]$} \\
\hline $\begin{array}{l}\text { Heard } \\
\text { deceased }\end{array}$ & 980 & $\begin{array}{l}\text { Yes } \\
\text { (no/unsure) }\end{array}$ & 0.82 & 2.01 & .15 & {$[0.63,1.07]$} \\
\hline $\begin{array}{l}\text { Touched } \\
\text { deceased }\end{array}$ & 495 & $\begin{array}{l}\text { Yes } \\
\text { (no/unsure) }\end{array}$ & 1.67 & 5.91 & .01 & {$[1.10,2.54]$} \\
\hline $\begin{array}{l}\text { Smelled } \\
\text { deceased }\end{array}$ & 976 & $\begin{array}{l}\text { Yes } \\
\text { (no/unsure) }\end{array}$ & 0.92 & 0.26 & .60 & {$[0.68,1.24]$} \\
\hline $\begin{array}{l}\text { Received } \\
\text { message }\end{array}$ & 976 & $\begin{array}{l}\text { Yes } \\
\text { (no/unsure) }\end{array}$ & 0.83 & 1.74 & .18 & {$[0.63,1.09]$} \\
\hline
\end{tabular}




\begin{tabular}{|c|c|c|c|c|c|c|}
\hline Pre dictor & $N$ & $\begin{array}{l}\text { Category } \\
\text { (reference) }\end{array}$ & $O R$ & Wald $_{1}$ & $p$ & $95 \%$ CI for $O R$ \\
\hline $\begin{array}{l}\text { Sensed } \\
\text { pre s e n c e of } \\
\text { deceased }\end{array}$ & 981 & $\begin{array}{l}\text { Yes } \\
\text { (no/unsure) }\end{array}$ & 0.94 & 0.14 & .70 & {$[0.71,1.25]$} \\
\hline Crisis ADC & 982 & $\begin{array}{l}\text { Yes } \\
\text { (no/unsure) }\end{array}$ & 0.94 & 0.10 & .74 & {$[0.68,1.31]$} \\
\hline $\begin{array}{l}\text { Frightened by } \\
\text { ADC }\end{array}$ & 982 & $\begin{array}{l}\text { Yes } \\
\text { (No/unsure) }\end{array}$ & 1.56 & 5.15 & .02 & {$[1.06,2.31]$} \\
\hline $\begin{array}{l}\text { Sentiment about } \\
\text { ADC }\end{array}$ & 982 & $\begin{array}{l}\text { Positive } \\
\text { (negative/neutral) }\end{array}$ & 1.23 & 0.71 & .39 & {$[0.75,2.02]$} \\
\hline $\begin{array}{l}\text { Knew the } \\
\text { deceased }\end{array}$ & 977 & $\begin{array}{l}\text { Yes } \\
\text { (no/unsure) }\end{array}$ & 1.49 & 2.47 & .11 & {$[0.90,2.48]$} \\
\hline $\begin{array}{l}\text { Comfort from } \\
\text { ADC }\end{array}$ & 983 & $\begin{array}{l}\text { Yes } \\
\text { (no/unsure/N/A) }\end{array}$ & 1.61 & 8.68 & .003 & {$[1.17,2.21]$} \\
\hline
\end{tabular}

$O R=$ Odds ratio; Wald $_{1}=$ Wald statistic with 1 degree of freedom; $p=\mathrm{p}$-value; $\mathrm{CI}=$ confidence interval.

Table 10. Multivariate Analysis Predicting Beliefs About Deceased Persons Can Contact the Living Persons Pre- and Post-ADC (Binary Logistic Regression), N = 488.

\begin{tabular}{|l|l|l|l|l|l|}
\hline Predictor & $\begin{array}{l}\text { Category } \\
\text { (reference) }\end{array}$ & OR & Wald 1 & $P$ & $95 \%$ CI for OR \\
\hline Education & - & 0.80 & 5.70 & 0.01 & {$[0.66,0.96]$} \\
\hline Multiple ADCs & Yes (no/unsure) & 0.61 & 3.62 & 0.05 & {$[0.36,1.01]$} \\
\hline Touched deceased & Yes (no/unsure) & 1.83 & 7.33 & 0.007 & {$[1.18,2.84]$} \\
\hline $\begin{array}{l}\text { Frightened by } \\
\text { ADC }\end{array}$ & Yes (no/unsure) & 2.07 & 6.31 & 0.02 & {$[1.17,3.66]$} \\
\hline $\begin{array}{l}\text { Comfort from } \\
\text { ADC }\end{array}$ & $\begin{array}{l}\text { Yes } \\
\text { (no/unsure/N/A) }\end{array}$ & 1.71 & 4.73 & 0.03 & {$[1.05,2.79]$} \\
\hline
\end{tabular}

$O R=$ Odds ratio; Wald $_{1}=$ Wald statistic with 1 degree of freedom; $p=\mathrm{p}$-value; $\mathrm{CI}=$ confidence interval.

Regarding spirituality, participants who endorsed an ADC with physical contact showed a 51\% increase in odds of strengthening their spiritual beliefs post-ADC, compared with those who reported no physical contact experience. In addition, endorsing experiencing an ADC at the moment of death of the perceived person (a "crisis ADC") and an ADC that brings comfort trended toward significance in enhancing spirituality, although they did not reach statistical significance.

Our research further supports that trying to touch the deceased, receiving a message from the deceased, or being frightened by the contact from the deceased were significant moderating factors in increased belief in life after death post-ADC. Regarding change in beliefs in life after death, 
participants who i) endorsed trying to touch the deceased showed $89 \%$ increased, ii) received a message from the deceased showed $41 \%$ decreased, and iii) endorsed being frightened by the ADC showed $157 \%$ increased odds in believing in life after death after their ADC than those who did not endorse such experiences.

When examining beliefs about deceased persons being able to contact the living, those participants who i) tried to touch, ii) were frightened by the contact with the deceased and iii) perceived ADC as comforting or emotionally healing showed higher odds or increased belief post-ADC versus those participants who did not report such experiences (see Tables 3 to 10).

\section{Discussion}

ADCs occur and are generally experienced as meaningful in a positive way. The majority of participants in our study endorsed that their ADC was 'important', or even 'life changing' and only $12.1 \%$ of the participants reported their ADC experience as frightening, which is very comparable with previous research in the area. Belief in life after death and the fact that the deceased can communicate with the living also significantly increased in participants after their reported ADC. Finally, experiencing an ADC appears to have a significant impact on reducing fear of death in our participants. This is consistent with much of the existing literature, which proposes that ADCs may serve to provide an ongoing connection to the deceased and suggests to those who are living that death is not final, thus presumably reducing fear of death (Keen et al., 2013; Kwilecki, 2011; Steffen \& Coyle, 2011). Although this may be theoretically plausible, identification of the causes for reductions in fear of death cannot be discerned with the current data, and additional research exploring the causal thought processes of individuals who experience and ADC would be beneficial.

According to previous research, it is not unusual for those who report ADCs to not only experience a reduced fear of death, but also develop an increased interest in spirituality as well as the exploration of existential issues about God and the universe via religion (Streit-Horn, 2013). AQ2 However, Barbato (1999) has previously reported no correlation between the occurrence of parapsychological experiences such as ADCs and endorsed religious, spiritual, or cultural status. The findings of our study suggest that ADCs tend to be associated with increased spirituality beliefs but they have less impact on religiosity beliefs (Barbato et al., 1999). Interestingly, our participants who endorsed an ADC with physical contact were more likely to endorse increased spirituality than participants who reported no physical contact with their ADCs. The only factor that appeared to be significantly associated with increased religiosity in our study was having multiple ADCs. Rather than frequency, it has been shown in prior research that religious, spiritual, and cultural beliefs tend to influence the content and interpretation of ADCs (Datson \& Marwit, 1997; Lange \& Houran, 1997; Rees, 1971). In studies by Keen et al. (2013) most participants reported using religious or spiritual beliefs to explain and understand their experiences of ADCs (Keen et al., 2013). It is unclear from the literature why 
having multiple ADCs may be associated with increased religiosity, and additional information regarding specific religious beliefs may need to be captured to more fully understand this finding.

When exploring moderating factors in belief in life after death after experiencing an ADC, we found that trying to touch the deceased, receiving a message from the deceased, or being frightened by the contact from the deceased all were significant. One can speculate that the increased salience of such experiences during an ADC could have an impact on belief in life after death. The physical or emotional components associated with such characteristics of an experienced ADC may help promote or solidify the belief in life after death more than other associated characteristics such as sense of presence. Simply put, we may have a paranormal experience in the form of an ADC but our physical sensations also inform our interpretation of it and the impact it may have upon our beliefs. Similarly, beliefs about the deceased contacting the living were significantly impacted by trying to touch the deceased, being frightened by the contact, and perceiving the ADC as healing or comforting. Thus perceived physical contact or emotions such as fear and comfort may inform our concept and understanding of an experienced ADC and thus help change our beliefs regarding the ability of deceased to contact the living.

\section{Study Limitations}

Research on spontaneous ADCs is challenging for multiple reasons including the obvious fact that one cannot predict when an ADC will occur, and it is not possible to construct a typical randomized controlled trial to test for their impacts (Hastings et al., 2002; Moody, 1992). There is certainly work in the area of what may be considered "induced ADCs" via use of a psychomanteum or other methodology to help facilitate contact with the deceased (Hastings et al., 2002; Moody, 1992), but this may be considered slightly different than what we explored in this study, which is a spontaneous ADC. Thus, in this study, we must depend on accurate self-reported information from participants regarding their ADC as well as their beliefs and thoughts both before and after the reported ADCs. This is obviously a limitation of the current research since we are depending on potentially flawed or incomplete recall of experiences, thoughts, beliefs, and emotions, which may have occurred many years ago. We do not have the space in this article to dive into the details of memory, emotion, and recall, but encourage interpretation of the current self-reported data with caution.

An additional limitation is the reticence of some individuals to share ADC experiences (Streit-Horn, 20 13). Individuals who decided to share their experience on our survey may be different in some important way from other individuals who have experienced an ADC and chose not to share it. Individuals who decide not to share may be worried about being negatively judged for sharing such information or may have some other fear related to sharing. Some individuals may not wish to share such an intimate experience with others. Some individuals may have perceived the experience in a negative light, or found it too distressful or disturbing to discuss. Thus, it is a very real possibility that 
our sample is unique and not representative of all who experience an ADC. Without additional information about that specific subpopulation, it is impossible to know if we have a representative sample in our current study although the large size of the sample partly mitigates this limitation. Another limitation of our current study is that although it is international in scope, it is not a comprehensive representation of much of the world. The survey was provided so far in only a handful of languages and thus, we cannot say that the data is representative of other cultures, but only of the samples surveyed. Additionally, data in the current study were gathered without the ethnicity information of the participants. Obviously, effect of ADC on beliefs is a broad structure encompassing a number of different dimensions, possibly including ethnicity. As a result, the effects of ethnicity on belief and spirituality changes related to ADC could not have been addressed in this study. Again, more comprehensive data will need to be collected in order to promote a fuller representation of the human experiences of ADCs.

\section{Future Research}

What does appear to be evident is that the overwhelming reaction to having an ADC is positive. This is demonstrated in existing research literature and replicated in the current research. Previous research has demonstrated that sharing the ADC can be beneficial for the individual experiencing it (Streit-Horn , 2013). Thus, future research and clinical work in this area may benefit from focusing on the beneficial effects of experiencing and sharing ADCs (Roxburgh \& Evenden, 2016a, 2016b). This would include adding research questions about participants' previous disclosures, as well as their thoughts and feelings regarding the ADC. Besides participant observation, future research may focus on the relationship of deceased with the participant, the severity of grief, subgroups who experience ADC during a traumatic grief period. Exploring these parameters would show us the intersection of experiencing ADC with factors related to the deceased, properties of the mourning process, and effect of post-traumatic growth. Additionally, information about the cultural acceptance of ADC would be of interest to collect and analyze. Training clinical professionals on how to ask about ADCs as well as how to listen with openness and acceptance in order to help those who have ADCs accommodate their experiences is an additional area of future research (Amatuzio \& Matthews, 2007; Hastings, 1983; Wo oten-Green, 2001). The benefits of wider acknowledgement and improved understanding of this fairly prevalent and positive experience appears to be an important topic and thus a logical and helpful focus for future research.

Declaration of Conflicting Interests The author(s) declared no potential conflicts of interest with respect to the research, authorship, and/or publication of this article.

FundingThe author(s) received no financial support for the research, authorship, and/or publication of this article. 
ORCID iD Jennifer K. Penberthy https://orcid.org/0000-0001-5717-2231, Marieta Pehlivanova https://orcid.org/0000-0002-3853-1267, Tevfik Kalelioglu https://orcid.org/0000-00019319-055X

\section{References AQ3}

Amatuzio, J., \& Matthews, R. (2007). Beyond knowing: Mysteries and messages of death and life from a forensic pathologist. Journal of Nuclear Medicine, 48(10) , 1749-1749. https://doi.org/10.2967/jnumed.107.043562

Arcangel, D. (2005). Afterlife encounters: Ordinary people, extraordinary experiences. Hampton Roads.

Barbato, M., Blunden, C., Reid, K., Irwin, H., \& Rodriguez, P. (1999, June 8). Parapsychological phenomena near the time of death. Journal of Palliative Care-15(2):30-37. Advance online publication. https://doi.org/10.1177/082585979901500206

Beischel, J., Mosher, C., \& Boccuzzi, M. (2015). The possible effects on bereavement of assisted afte r-death communication during readings with psychic mediums: A continuing bonds perspective. OMEGA—Journal of Death and Dying, 70(2), 169-194. https://doi.org/10.2190/OM.70.2.b

Burton, J. (1982). Contact with the dead: A common experience? Fate, 35(4), 65-73.

Castelnovo, A., Cavallotti, S., Gambini, O., \& D’Agostino, A. (2015, August 11). Post-bereavement hallucinatory experiences: A critical overview of population and clinical studies. Journal of Affective Disorders. Advance online publication. https://doi.org/10.1016/j.jad.2015.07.032

Datson, S. L., \& Marwit, S. J. (1997). Personality constructs and perceived presence of deceased loved ones. Death Studies, 21(2), 131-146. https://doi.org/10.1080/074811897202047

Devers, E. (1997). Goodbye again: Experiences with departed loved ones. Kansas City, MO: Univer sal Press Syndicate. AQ4

Elsaesser, E., Roe, C., Cooper, C., \& Lorimer, D. (2021). The phenomenology and impact of hallucinations concerning the deceased. British Journal of Psychiaty Open, ๑

Guggenheim, B., \& Guggenheim, J. (1995). Hello from heaven! Bantam.

Haraldsson, E. (2006). Popular psychology, belief in life after death and reincarnation in the Nordic countries, Western and Eastern Europe. Nordic Psychology, 58(2) , 171-180. https://doi.org/10.1027/1901-2276.58.2.171 
Haraldsson, E. (2012). Cases of the reincarnation type and the mind-brain relationship.-In relationshipExploring frontiers of the mind-brain (pp. 215-231).In: Moreira-Almeida A., Santana San tos F. (eds) Exploring Frontiers of the Mind-Brain Relationship. Mindfulness in Behavioral Health. Springer. https://doi.org/10.1007/978-1-4614-0647-1_11 AQ5

Hastings, A. (1983) . A counseling approach to parapsychological experience. The Journal of Transpersonal Psychology, 15(2), 143.

Hastings, A. (2012). Effects on bereavement using a restricted sensory environment (psychomanteum) . Journal of Transpersonal Psychology, 44(1), 1.

Hastings, A., Hutton, M., Braud, W., Bennett, C., Berk, I., Boynton, T., Steinbach-Humphrey, S. (2002). Psychomanteum research: Experiences and effects on bereavement. OMEGA-Journal of Death and Dying, 45(3), 211-228. https://doi.org/10.2190/LV5G-E3JV-6CVT-FKN5

Houck, J. A. (2005). The universal, multiple, and exclusive experiences of after-death communication. Journal of Near-Death Studies, 24(2), 117-127 p. ". https://doi.org/10.17514/jnds-2005-24-2-p117-127 AQ6

Kalish, R. A., \& Reynolds, D. K. (1973). Phenomenological reality and post-death contact. Journal for the Scientific Study of Religion, 12(2), 209. https://doi.org/10.2307/1384890

Keen, C., Murray, C. D., \& Payne, S. (2013). A qualitative exploration of sensing the presence of the deceased following bereavement. Mortality, 18(4) 339-357. https://doi.org/10.1080/13576275.2013.819320

Krause, N. (2011). Reported contact with the dead, religious involvement, and death anxiety in late life. Review of Religious Research, 52(4), 347-364.

Kwilecki, S. (2011). Ghosts, meaning, and faith: After-death communications in bereavement narrativ es. Death Studies, 35(3), 219-243. https://doi.org/10.1080/07481187.2010.511424

La Grande, L. E. (1997). After death communication: Final farewells. Llewellyn.

La Grande, L.E. (1999). Messages and miracles: Extraordinary experiences of the bereaved. Llewellyn.

Lange, R., \& Houran, J. (1997). Context-induced paranormal experiences: Support for Houran and Lange's model of haunting phenomena. Perceptual and Motor Skills, 84(3 PART II), 1455-1458. https://doi.org/10.2466/pms.1997.84.3c.1455

Long, J. P. (1999). After death communication overview. After Death Communication Research Fou 
ndation.

Moody, R. A. (1992). Family reunions: Visionary encounters with the departed in a modern-day psych omanteum. Journal of Near-Death Studies, 11(2), 83-121. https://doi.org/10.1007/BF01074301

Olson, P. R., Suddeth, J. A., Peterson, P. J., \& Egelhoff, C. (1985). Hallucinations of widowhood. Journal of the American Geriatrics Society, 33(8) , 543-547. https://doi.org/10.1111/j.15325415.1985.tb04619.x

Rees, W. D. (1971). The hallucinations of widowhood. British Medical Journal 4(5778), 37-41. https://doi.org/10.1136/bmj.4.5778.37

Ring, K. (2008). The death and posthumous life of Tom Sawyer: A case study of apparent after-death communication. Journal of Near-Death Studies, 27(2), 111-133 ". https://doi.org/10.17514/jnds-200827-2-p111-133 AQ7

Roxburgh, E.C., \& Evenden, R.E. (2016a). 'Most people think you're a fruit loop': Clients' experiences of seeking support for anomalous experiences. Counselling and Psychotherapy Research, 16(3), 211-221. https://doi.org/10.1002/capr.12077

Roxburgh, E. C., \& Evenden, R. E. (2016b). 'They daren’t tell people': Therapists' experiences of working with clients who report anomalous experiences. European Journal of Psychotherapy and Counselling, 18(2), 123-141. https://doi.org/10.1080/13642537.2016.1170059

Sormanti, M., \& August, J. (1997). Parental bereavement: Spiritual connections with deceased children. American Journal of Orthopsychiatry, 67(3), 460-469. https://doi.org/10.1037/h0080247

Steffen, E., \& Coyle, A. (2011). Sense of presence experiences and meaning-making in bereavement: A qualitative analysis. Death Studies, 35(7), 579-609. https://doi.org/10.1080/07481187.2011.584758

Streit-Horn, J. (2013). A systematic review of research on after-death communication (ADC). Disserta tion Abstracts International: Section B: The Sciences and Engineering. AQ8

Woollacott, M., Roe, C. A., Cooper, C. E., Lorimer, D., \& Elsaesser, E. (2021). Perceptual phenome na associated with spontaneous experiences of after-death communication: Analysis of visual, tactile, a uditory and olfactory sensations. Explore. Advance online publication. https://doi.org/10.1016/j.explore.2021.02.006

Wooten-Green, R. (2001). When the dying speak: How to listen to and learn from those facing death. Loyola. 


\section{Author Biographies}

Jennifer K. Penberthy, Ph.D., ABPP is the Chester F. Carlson Professor of Psychiatry and Neurobehavioral Sciences at the University of Virginia School of Medicine. She is a board certified licensed clinical psychologist and conducts research and clinical work in the area of contemplative sciences and consciousness. - AQ9

Marieta Pehlivanova -is a researcher at the Division of Perceptual Studies at the University of Virginia. Her work at the Division focuses on near-death experiences, children reporting past-life memories, as well as other unusual experiences. Marieta holds a doctorate in experimental psychology and a bachelor's degree in statistics. Prior to her academic work, she pursued a career as a statistician in medical research.

Tevfik Kalelioglu, M.D., is a Research Assistant Professor at Psychiatry and Neurobehavioral Sciences at the University of Virginia School of Medicine."

Chris A. Roe holds a Chair in Psychology at the University of Northampton, UK, and is Director of the Centre for the Psychology and Social Sciences that is based there. He is the International Affiliate for England of the Parapsychology Foundation and is a Vice-President of the Society for Psychical Research. -

Callum E. Cooper is a Chartered Member of the British Psychological Society and a Council Member of the Society for Psychical Research. He is a senior lecturer in psychology at the University of Northampton and co-ordinator of the 3rd year module in Parapsychology \& Anomalous Experiences.

\section{David Lorimer *}

Evelyn Elsaesser is an independent researcher and author in the field of death-related experiences, notably Near-Death Experiences (NDEs) and After-Death Communications (ADCs). She is the project leader of the research project "Investigation of the Phenomenology and Impact of Spontaneous AfterDeath Communications (ADCs) and Founding member and member of the Scientific Committee of Swiss IANDS. She lives in Switzerland.* 


\section{Author Query}

1. Query: [AQ1] - Please provide complete reference details for "Datson-Marwit et al. (1997)"

Answered within text

2. Query: [AQ2] - Please provide complete reference details for "Barbato (1999)."

Answered within text

3. Query: [AQ3] - Journal style is to list all authors when there are 20 or fewer; when 21 or more, first 19 should be listed and then ellipses followed by last author should be added. If 20 or fewer, please list all the authors and if 21 or more, list first 19 and then ellipses followed by last author.

Ok

4. Query: [AQ4] - Please provide publisher details for "Devers, E. (1997)." Answered within text

5. Query: [AQ5] - Please provide editors name, if any, for reference "Haraldsson, E. (2012)." Answered within text

6. Query: [AQ6] - Please provide page range for reference "Houck, J. A. (2005)." Answered within text

7. Query: [AQ7] - Please provide page range for reference "Ring, K. (2008)." Answered within text

8. Query: [AQ8] - Please provide volume and page range for reference "Streit-Horn, J. (2013)."

Since this is a dissertation thesis, it doesn't have a volume or page number

9. Query: [AQ9] - Please provide brief biography for all authors.

Ok

\section{General Query}

1. Query: GQ1: Please confirm that all author information, including names, affiliations, sequence, and contact details, is correct. 


\section{Response [ role="Author" tevfikkaleli@hotmail.com]: Ok}

2. Query: GQ2: Please review the entire document for typographical errors, mathematical errors, and any other necessary corrections; check headings, tables, and figures.

\section{Response [ role="Author" tevfikkaleli@hotmail.com]: Ok}

3. Query: GQ3: Please confirm that the Funding and Conflict of Interest statements are accurate.

Response [ role="Author" tevfikkaleli@hotmail.com]: Ok

4. Query: GQ4: Please confirm you have reviewed this proof to your satisfaction and understand this is your final opportunity for review prior to publication.

Response [ role="Author" tevfikkaleli@hotmail.com]: Ok 